\title{
GROUND MOTION SELECTION FOR SIMULATION-BASED SEISMIC HAZARD AND STRUCTURAL RELIABILITY ASSESSMENT
}

\author{
Brendon A Bradley ${ }^{*}$, Lynne S Burks, Jack W Baker \\ *corresponding author: brendon.bradley@canterbury.ac.nz
}

\begin{abstract}
This paper examines four methods by which ground motions can be selected for dynamic seismic response analyses of engineered systems when the underlying seismic hazard is quantified via ground motion simulation rather than empirical ground motion prediction equations. Even with simulation-based seismic hazard, a ground motion selection process is still required in order to extract a small number of time series from the much larger set developed as part of the hazard calculation. Four specific methods are presented for ground motion selection from simulation-based seismic hazard analyses, and pros and cons of each are discussed via a simple and reproducible illustrative example. One of the four methods (method 1 'direct analysis') provides a 'benchmark' result (i.e. using all simulated ground motions), enabling the consistency of the other three more efficient selection methods to be addressed. Method 2 ('stratified sampling') is a relatively simple way to achieve a significant reduction in the number of ground motions required through selecting subsets of ground motions binned based on an intensity measure, IM. Method 3 ('simple multiple stripes') has the benefit of being consistent with conventional seismic assessment practice using as-recorded ground motions, but both methods 2 and 3 are strongly dependent on the efficiency of the conditioning IM to predict the seismic responses of interest. Method 4 ('GCIM-based selection') is consistent with 'advanced' selection methods used for as-recorded ground motions, and selects subsets of ground motions based on multiple IMs, thus overcoming this limitation in methods 2 and 3 .
\end{abstract}

\section{KEYWORDS}

Ground motion selection; seismic hazard analysis; ground motion simulation.

\section{INTRODUCTION}

The selection of ground motion time series provides the critical link between site-specific probabilistic seismic hazard analyses (PSHA) and dynamic seismic response analysis to quantify the seismic performance of a system of interest. Conventional PSHA employs empirical ground motion prediction equations (GMPEs) [e.g. 1], which provide statistical predictions of ground motion intensity measures (IMs) based on regression analysis of historical ground motion observations. Because dynamic seismic response analyses require a ground motion time series as an input excitation, a key goal of conventional ground motion selection is the determination of ground motion ensembles which are representative of the theoretical (or 'target') distribution of one of more ground motion IMs for the seismic hazard considered [25].

Research regarding physics-based simulation for ground motion prediction is 
rapidly advancing as a result of increases in the understanding of ground motion physics [6,7], computational capabilities [8], and the observed diminishing returns from incremental advances in empirical GMPEs [9]. In particular, the Cybershake project [8] makes use of hybrid broadband ground motion simulations based on kinematic ruptures to conduct PSHA for long-period response spectral ordinates in the Southern California region. Due to the consideration of multiple slip distributions and hypocenter locations for each rupture, and numerous potential ruptures, one PSHA curve in the Cybershake project comprises typically 415,000 ground motion time series [8].

Ground motion simulation may, on first glance, seem to circumvent the need for ground motion selection because such methods provide a ground motion time series directly. However, for seismic response analysis a limited number of ground motion excitations are typically desired (typically on the order of 100 for a comprehensive multi-objective performance assessment, but as low as 7 for some single-objective design standard prescriptions, or exceeding several hundred for research-based analyses). Additionally, a simulation-based approach will produce many more lowamplitude ground motions than high-amplitude ones (because the former occur more often in nature), while engineering assessments are typically focused on high amplitude shaking likely to cause damage. The need to select a small number of ground motions from a large set of simulations, and the greater importance of higheramplitude motions indicate that ground motion selection methods are still required for simulation-based SHA in order to determine a representative subset of motions for use in seismic response analysis of engineered systems.

This paper presents four approaches for ground motion selection from simulation-based SHA along with a discussion of their relative advantages and disadvantages through a simple and reproducible example. The estimation of ground motion hazard through simulation-based SHA and its parallels with GMPE-based SHA is first presented. Details of the illustrative case study are then outlined, followed by the presentation of each of the four ground motion selection methods and salient features of their application. Finally, the relative pros and cons of each method in relation to accuracy, precision, and computational demands are presented.

\section{ESTIMATION OF GROUND MOTION HAZARD}

Ground motion hazard is most commonly quantified via probabilistic seismic hazard analysis (PSHA) [10, 11], and simulation-based PSHA will be the focus herein. However, brief comment is made at the end of this paper on the (more simple) application of simulation-based ground motion prediction and selection for scenariobased SHA.

PSHA accounts for both the likelihoods of various earthquake rupture occurrences and the distribution of some ground motion intensity measure (IM) for each earthquake rupture, which mathematically can be expressed as:

$$
\lambda_{I M}(\mathrm{im})=\sum_{k=1}^{N_{\text {rup }}} P_{I M \mid R u p}\left(\operatorname{im} \mid r u p_{k}\right) \lambda_{R u p}\left(\operatorname{rup}_{k}\right)
$$

where $\lambda_{I M}(\mathrm{im})$ is the seismic hazard curve providing the (mean) annual rate of $I M>i m ; P_{I M \mid R u p}\left(\right.$ im $\left._{\text {rup }}\right)$ is the probability that $I M>i m$ given earthquake rupture Rup $=\operatorname{rup}_{k} ; \lambda_{R u p}\left(\operatorname{rup}_{k}\right)$ is the annual rate of $r u p_{k}$; and $N_{\text {rup }}$ is the number of earthquake ruptures posing a hazard at the site (as defined by an earthquake rupture 
forecast, ERF [12]).

It should be noted that $P_{I M \mid R u p}\left(\right.$ im $\left._{r u p}\right)$ can be estimated from either empirical ground motion prediction equations or from ground motion simulations. In the case of GMPEs, $P_{I M \mid R u p}\left(\right.$ im $\left._{\text {rup }}\right)$ is obtained from the GMPE-computed mean and standard deviation and an assumed lognormal distribution assumption [13], while for ground motion simulations it can be obtained in a similar parametric form, or alternatively as the fraction of simulations from $\operatorname{Rup}=\operatorname{rup}_{k}$ that produce time series with $I M>\mathrm{im}$. For direct comparison with GMPEs, a rupture may be defined as slip on a fault with a specified geometry and an average focal mechanism, such that there is uncertainty in the hypocenter location, slip distribution, and temporal evolution of slip on the fault (i.e. the kinematic rupture parameters). Uncertainty in these variables for $\operatorname{Rup}=\operatorname{rup}_{k}$ can then be sampled via $N_{k}$ Monte Carlo simulations, so that $P_{I M \mid R u p}\left(\right.$ im $\left.\mid r u p_{k}\right)$ is obtained from:

$$
P_{I M \mid R u p}\left(\operatorname{im} \operatorname{rup}_{k}\right)=\frac{1}{N_{k}} \sum_{n=1}^{N_{k}} I\left(I M>\operatorname{im}_{\operatorname{rup}} \operatorname{rup}_{k, n}\right)
$$

where $I\left(I M>\operatorname{im} \mid r u p_{k, n}\right)$ is an indicator function equal to one if $I M>i m$ for the $n^{\text {th }}$ Monte Carlo simulation of $\operatorname{rup}_{k}$, and zero otherwise.

An alternative to the rupture definition in the above seismic hazard formulation is to consider each Monte Carlo simulation of $\operatorname{rup}_{k}$ as a unique rupture in itself, $\operatorname{rup}_{i}$, with an annual rate of occurrence of $\lambda_{i}=\lambda_{R u p}\left(\operatorname{rup}_{k}\right) / N_{k}$, in which case the seismic hazard can be computed from:

$$
\lambda_{I M}(\mathrm{im})=\sum_{i=1}^{N_{\text {rup }}^{*}} I\left(I M>\text { im } \mid \text { rup }_{i}\right) * \lambda_{i}
$$

where $N_{\text {rup }}^{*}$ is the total number of these alternatively-defined ruptures (if $N_{k}$ was constant for all $\operatorname{rup}_{k}$ then $N_{\text {rup }}^{*}=N_{\text {rup }} * N_{k}$ ). The four subsequently considered ground motion selection methods lend themselves more favorably to one of the above two formats for representing the seismic hazard.

\section{CASE STUDY}

To illustrate the application of several ground motion selection strategies, a case-study is considered here using a specific ERF and site of interest, an algorithm for ground motion simulation from each earthquake rupture in the ERF, and a seismic response model to examine the impact of the selected ground motions on seismic demands.

The site considered is located in urban Christchurch, New Zealand (Lat: 43.5300; Lon: 172.6203) on soft soils with a 30m-averaged shear wave velocity, $V_{s 30}=200 \mathrm{~m} / \mathrm{s}$. The seismic rupture sources are defined by the current NZ ERF of Stirling et al. [14]. Although not required in general, seismic hazard analyses were first performed using empirical GMPEs to identify the 100 earthquake ruptures which contribute most significantly to the seismic hazard (the ERF of Stirling et al. [14] contains ruptures from over 500 fault and 20,000 distributed seismicity sources, the majority of which pose negligible hazard at the site). For each of these $N_{\text {rup }}=100$ ruptures, $N_{k}=50$ ground motion simulations were performed to enable determination of the seismic hazard according to Equations (1)-(3) (i.e. a total of 5,000 ground motion simulations). Figure 1 illustrates the seismic hazard curve at the 
site for (pseudo) spectral acceleration at a vibration period of $T=1.32 \mathrm{~s}$ as the IM, as well as 10 annual rates of exceedance that are considered in ground motion selection Methods 2-4, which correspond to exceedance probabilities of $[80 \%, 50 \%, 20 \%, 10 \%$, $5 \%, 2 \%, 1 \%, 0.5 \%, 0.2 \%, 0.1 \%]$ in 50 years.

Ground motion simulations were produced using the algorithm of Yamamoto and Baker [15]. This is a stochastic-process-based simulation algorithm that produces single-component ground motion time series with amplitude and frequency nonstationarity. Properties of the ground motion are functionally dependent on the causal earthquake's magnitude and distance, and the site's $V_{s 30}$. The algorithm was empirically calibrated so that the simulations' IMs (e.g. response spectra, duration, Arias intensity) are consistent with corresponding GMPEs for shallow crustal earthquakes. This algorithm is used for illustration because of its simplicity and speed (so that the presented examples and data are transparent and easy for readers to reproduce themselves). In order to illustrate the statistical precision of the different ground motion selection methods, 10 independent replicates of the simulated ground motions were obtained, so that in total 50,000 ground motion simulations and subsequent seismic response analyses were performed.

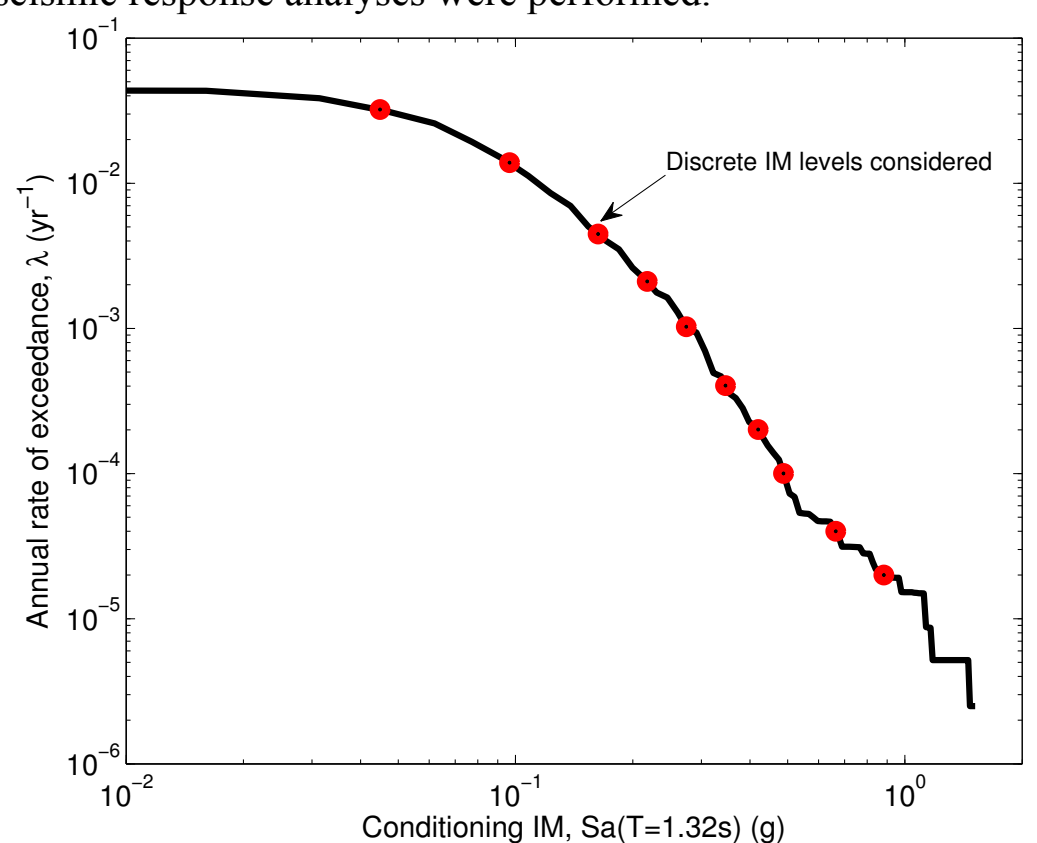

Figure 1: Seismic hazard curve for $\mathrm{Sa}(\mathrm{T}=1.32 \mathrm{~s})$ based on 5000 ground motion simulations at the site of interest.

The structure considered is a nonlinear degrading single-degree-of-freedom (SDOF) with an elastic period of $T=1.32$ seconds. Figure 2 illustrates the hysteretic backbone relationship of the SDOF, while unloading/reloading behavior was defined by the 'peak-oriented' relationship of Ibarra and Krawinkler [16]. Attention herein is given to the peak displacement of the SDOF mass. This structure is complex enough that simple ground motion IMs are not fully predictive of its response, but simple enough that it is feasible to perform thousands of analyses in order to obtain a robust 'benchmark' result to compare to. 


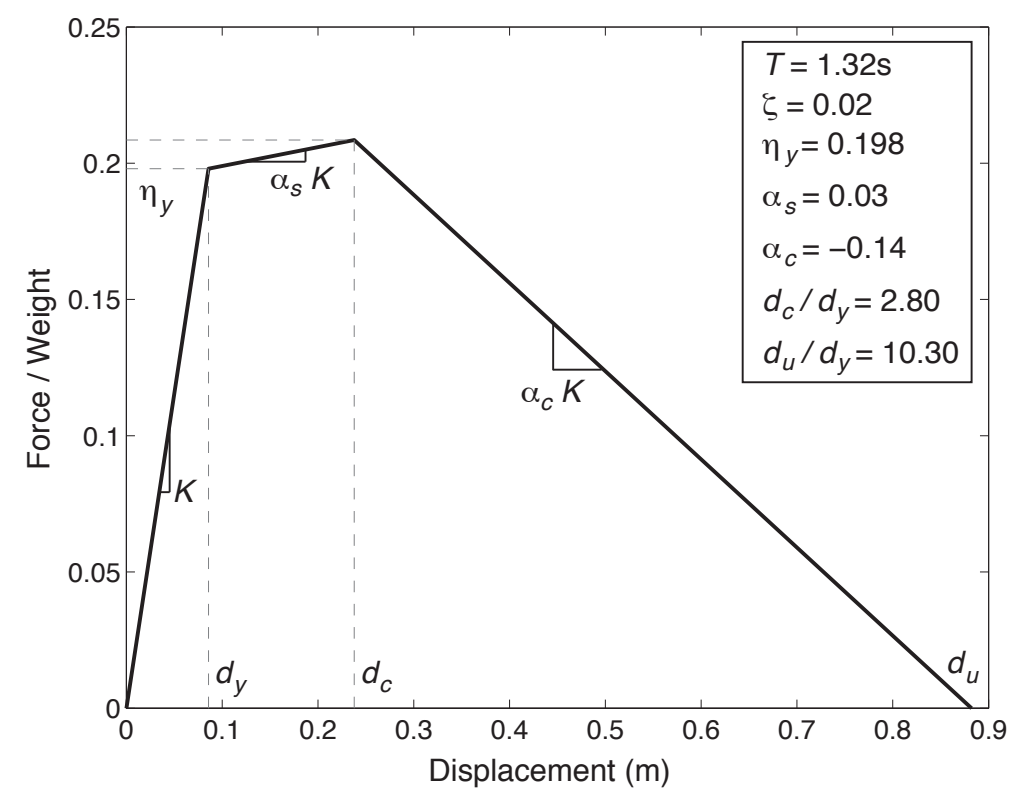

Figure 2: Hysteretic backbone of the inelastic SDOF system considered.

The ground motion selection procedures presented in the subsequent sections are also directly applicable for more complex structural models and ground motion simulation approaches. These less computationally expensive models are used here simply for convenience and clarity of illustration.

\section{GROUND MOTION SELECTION METHODS FOR SIMULATION-BASED SHA}

In this section, four approaches are presented by which simulation-based ground motions can be selected for engineering seismic response analyses. The results of the seismic response analyses are used to compute the seismic demand hazard (defined subsequently) for the peak SDOF displacement, however, where appropriate it is noted that other metrics for seismic performance quantification can also be obtained. Source codes for the implemented methods can be accessed via: http://purl.stanford.edu/wq807vm0009.

\section{Method 1: Direct Analysis}

In the 'direct analysis' method all simulated ground motions that are used to obtain the seismic hazard are used in seismic response analyses. Using this approach the annual rate of exceeding a specific level of seismic demand, EDP, can be mathematically written as:

$$
\lambda_{E D P}(e d p)=\sum_{i=1}^{N_{\text {rup }}^{*}} I\left(E D P>\operatorname{edp} \mid r u p_{i}\right) * \lambda_{i}
$$

where $\lambda_{E D P}(e d p)$ is the seismic demand hazard, providing the annual rate of $E D P>e d p$; and $I\left(E D P>e d p \mid r u p_{i}\right)$ is an indicator function equal to one if $E D P>e d p$ for the $\mathrm{i}^{\text {th }}$ rupture and zero otherwise. Note the calculation of $\lambda_{E D P}(e d p)$ in Equation (4) is very similar to $\lambda_{I M}(\mathrm{im})$ in Equation (3).

Figure 3 illustrates the seismic demand hazard curves that are obtained from the application of method 1 (i.e. Equation (4)) with 10 independent replicates. It is noted that the variability in the 10 replicates increases as the annual exceedance rate 
decreases - a result of the decreasing number of ground motion simulations for which $E D P$ is exceeded. Where global instability of the SDOF system was observed, and the analysis fails to converge, the EDP value is essentially infinite so that the seismic demand hazard curves are observed to 'flat-line' and produce an annual rate of global collapse, $\lambda_{C}[17,18]$. The global collapse rate can be directly computed as $\lambda_{C}=\sum_{i \in C} \lambda_{i}$ (i.e. the sum of the rates for ground motion's which cause collapse). For the 10 replicates considered here the number of ground motions which cause global collapse ranges from 9 to 18 (out of the 5,000 ground motion simulations), however because of the different rupture occurrence rates, $\lambda_{i}$, the collapse rate can vary by greater than this factor of 2 (the smallest collapse rate of $\lambda_{C}=3.5 * 10^{-7}$ is below the minimum shown y-axis value in Figure 3). The small absolute number of seismic response analyses that cause collapse means that this collapse rate is not very well constrained, and the implications of this for the examples presented will be subsequently discussed.

The seismic demand hazard obtained using Method 1 is 'exact' in the limit as the number of simulations considered approaches infinity ('exact' to the extent that the ground motion and seismic response simulation models represent reality). Furthermore, in comparison with the subsequent three methods, Method 1 does not require the use of a 'conditioning IM' and thus is also conceptually simple. However, it should be obvious that Method 1 is computational intensive and generally impractical to utilize with more complex multi-degree-of-freedom seismic response models. For example, the results in Figure 3 require 5,000 seismic response analyses for each of the 10 demand hazard curves, while application to simulation-based SHA's such as Cybershake [8] would require orders of magnitude more seismic response analyses than this. In addition to the high computational demands, Method 1 is also inefficient in the sense that the majority of the simulated ground motions result in small seismic demands that are not of engineering interest. Without the introduction of an IM to quantify ground motion severity there is no way to overcome this problem. Nonetheless, while not practically appealing, Method 1 does serve as a useful 'benchmark' against which the following three methods can be compared for the simple SDOF structure considered here.

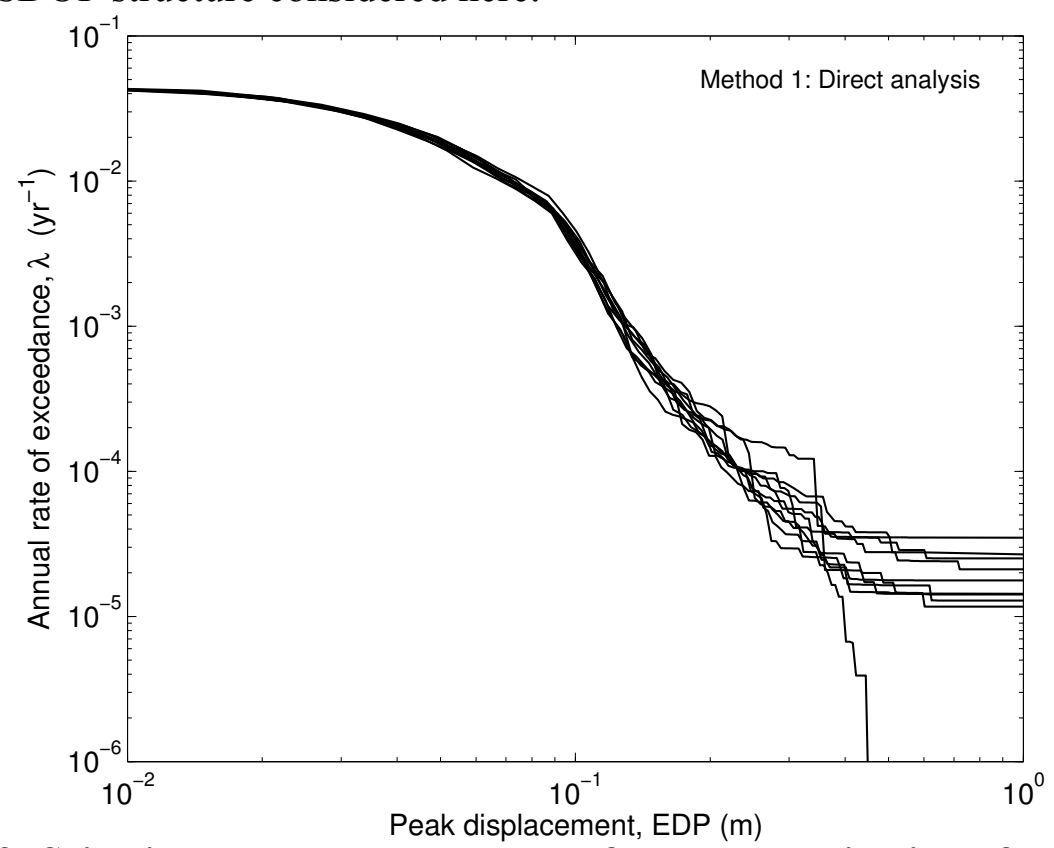

Figure 3: Seismic demand hazard curves from the application of Method 1. Each of the 10 replicate hazard curves utilizes 5,000 seismic response analyses. 


\section{Method 2: Stratified Sampling}

Stratified Sampling [e.g. 19] is a common approach in Monte Carlo simulation for addressing the computational efficiency problem with Method 1-that there are too many observations in the sample producing "uninteresting" results (i.e., low amplitude ground motions that produce small seismic demands). It is a widely used approach in structural reliability [42], and here we present its application to the selection of ground motions. The algorithm for stratified sampling in the context of simulation-based ground motion selection is as follows:

1. Select a conditioning $I M$ to quantify ground motion severity and define a discrete set of $n$ mutually exclusive bins for $I M$.

2. Within each IM bin, select $m$ ground motions (in proportion to their $\lambda_{i}$ values) for use in seismic response analyses

3. Reweight the annual rate of occurrence associated with each of the $m$ ground motions according to the equation:

$$
\lambda_{i, S S}=\lambda_{i} * \frac{\sum \text { all } \lambda_{i} \text { in bin } n}{\sum \text { all selected } \lambda_{i} \text { in bin } n}
$$

4. Compute the seismic demand hazard using Equation (4), with $\lambda_{i, S S}$ in place of $\lambda_{i}$, and the summation over only the seismic responses from the ground motions selected in the stratified sampling.

In order to demonstrate the application of steps 1 and 2 above, Figure 4 illustrates the use of stratified sampling to obtain the seismic responses from $m=10$ ground motions over $n=10$ different IM bins. The specific edges of the $I M$ bins were obtained from the mid-point between the $n=10$ different $I M$ levels depicted in Figure 1 (with equal half-widths used to define the outside edges of the two end bins). It can be seen that there are many more small amplitude ground motions that produce small seismic demands, and thus the use of stratified sampling helps to ensure that an appropiate number of the selected ground motions produce large seismic demands.

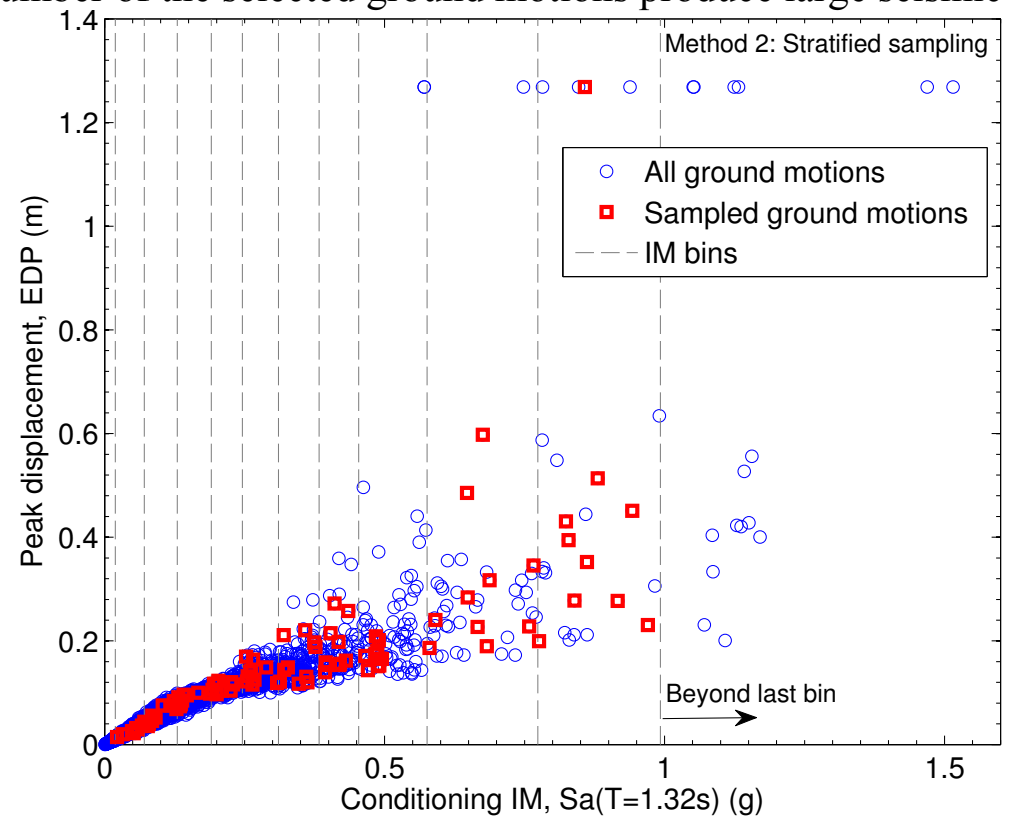

Figure 4: Illustration of stratified sampling used to quantify the relationship between EDP and IM through the selection of $m=10$ ground motions within $n=10$ different IM bins.

The selection of the conditioning $I M$ is a critical choice in Method 2, and the 
use of an IM that exhibits a weak correlation with the EDP of interest will result in greater uncertainty in the estimated seismic demand hazard (though the method is still unbiased in an average sense). Figure 5 provides an illustration of the use of stratified sampling with peak ground acceleration (PGA) as the conditioning $I M$, which has a weaker correlation with EDP than $I M=S a(T=1.32 \mathrm{~s})$. In the limit, where an $I M$ is used that exhibits no correlation with the $E D P$ of interest, stratified sampling over $n$ bins is statistically equivalent to random sampling over the entire set of simulations. Thus, as the 'efficiency' [20] of the IM decreases it becomes increasingly likely that the sampled ground motion simulations will be small in amplitude and therefore not of engineering interest.

Figure 6 illustrates the mean seismic demand hazard and its $80 \%$ confidence interval (CI) obtained from the application of Method 2 using $I M=S a(T=1.32 s)$ and $P G A$, as compared to the 'benchmark' result from Method 1. While the use of an $80 \% \mathrm{CI}$ is relatively non-standard, its adoption here is based on use of 10 replicates, meaning that the $10^{\text {th }}$ and $90^{\text {th }}$ percentiles used to define the $80 \%$ CI represent the $2^{\text {nd }}$ and $9^{\text {th }}$ sorted replicate values therefore minimizing the likelihood of statistical 'outliers' if a large CI was used. With exception of small EDP values (discussed further below), it can be seen that both applications of Method 2 result in CIs which encompass the mean from Method 1, thus illustrating that irrespective of the efficiency of the conditioning IM, Method 2 is unbiased. It is also apparent that the uncertainty in the seismic demand hazard, as reflected by the width of the CI, is notably larger for Method 2 than Method 1, with a larger CI when $I M=P G A$ because of its poorer efficiency with respect to $E D P$. It must be recalled however that this greater demand hazard uncertainty comes with the benefit of a significant reduction in the number of selected ground motions and consequent seismic response analyses: 5,000 analyses for Method 1, as compared to $n * m=100$ for each application of Method 2.

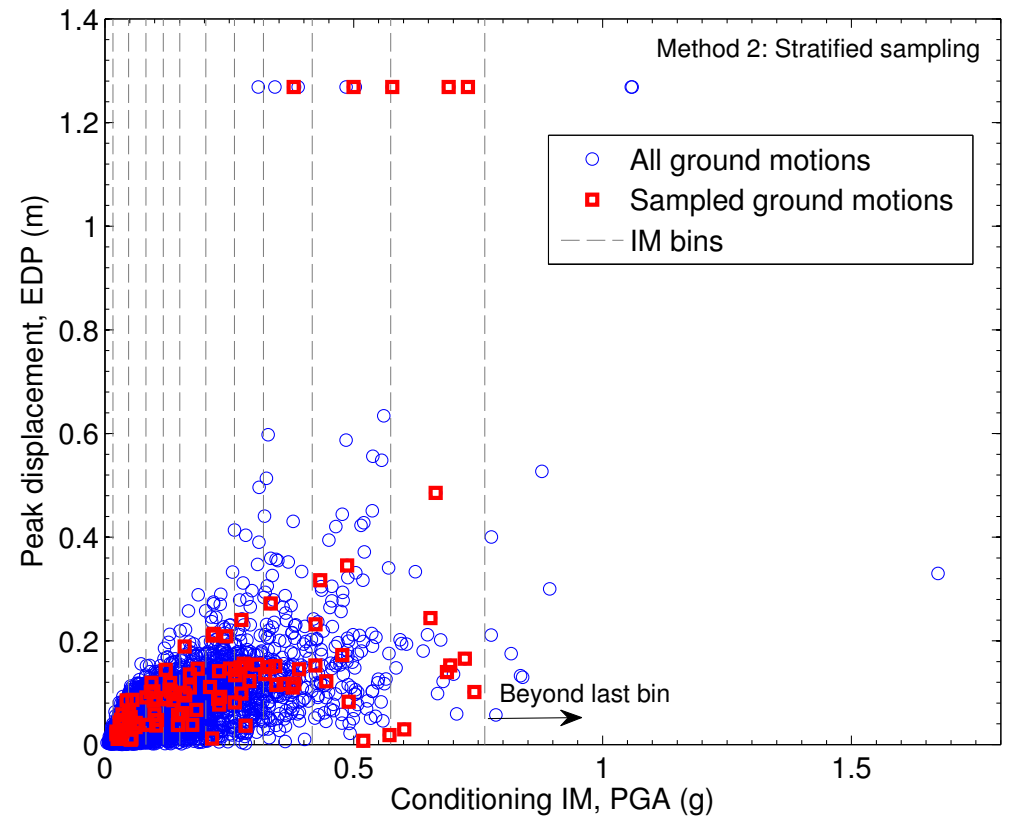

Figure 5: Illustration of stratified sampling using a conditioning $I M$ which is a poor predictor of the seismic demand (with $m=10$ ground motions per bin, and $n=10$ bins). 


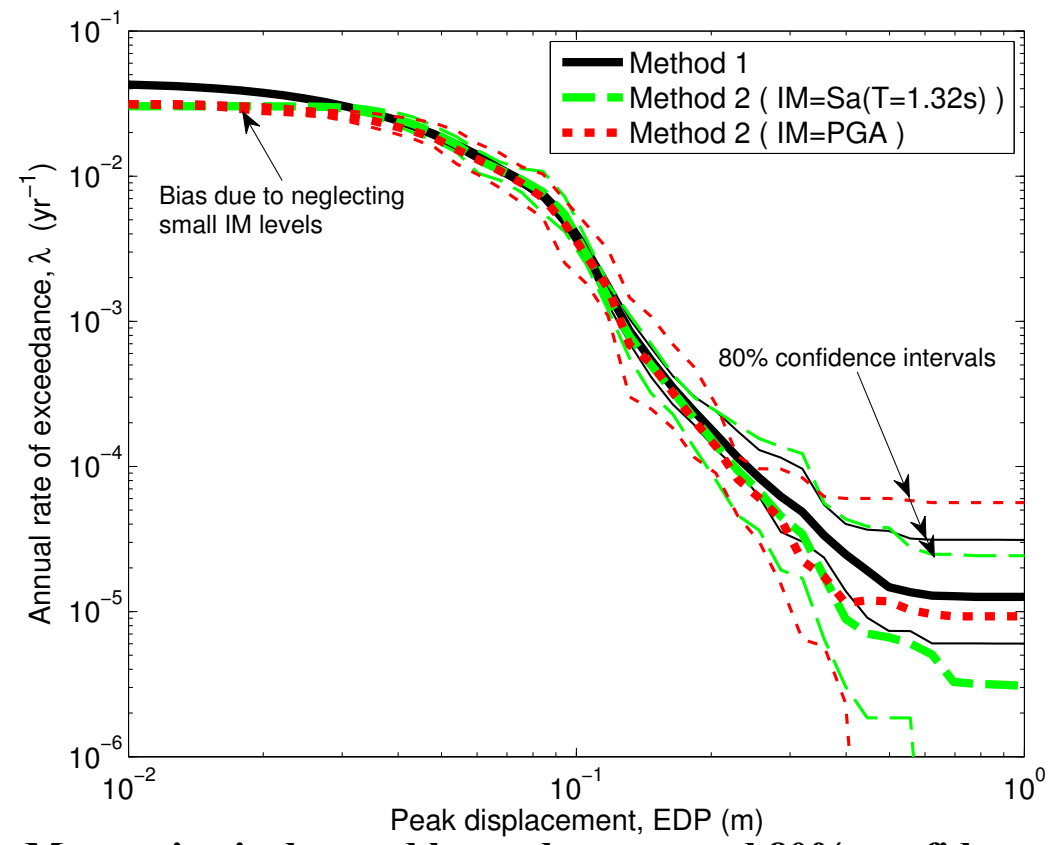

Figure 6: Mean seismic demand hazard curves and $80 \%$ confidence intervals from Method 2 using two different conditioning IMs in comparison with the results from Method 1. The bias for $\lambda>2.5 * 10^{-2}$ occurs because the smallest of the $n$ IM bins has a mid-point corresponding to a $70 \%$ exceedance probability in 50 years $\left(\lambda=2.4 * 10^{-2}\right)$.

While the value of an $I M$ having good correlation with the EDP of interest is relatively clear, selection of such an $I M$ in a practical setting is complicated by the fact that, for general seismic response analysis problems, there will be multiple EDPs of interest that are often sensitive to different aspects of the ground motion time series. As such, there is typically no single intensity measure that exhibits a strong correlation with multiple EDPs for non-trivial engineering systems [21]. In principle, stratified sampling can be applied to multiple IM parameters as well (e.g., one could build a grid of $\mathrm{Sa}(\mathrm{T}=1.32 \mathrm{~s})$ and PGA amplitudes, and select ground motions from each square in that grid). This may further extend the statistical benefits of the stratified sampling, though added complexity and the curse of dimensionality [22] will become significant if too many parameters are considered.

In summary, Method 2: stratified sampling has the benefit of being relatively simple, yet producing a potentially substantial reduction in the required number of seismic response analyses to compute the seismic demand hazard through preferentially selecting ground motions with the desired severity (via the conditioning IM). The benefit is greatest when the IM exhibits a strong correlation with the EDP of interest. The stratified sampling approach differs markedly from the conventional forms of seismic performance assessment, such as 'intensity-based' assessments [2328] (elaborated upon in the discussion of Method 3), and this may inhibit its initial use given that such procedures are the basis of current performance assessments within seismic design standards [e.g. 24, 25-27].

\section{Method 3: Simple Multiple Stripes Selection}

One of the practical issues alluded to in Method 2: Stratified sampling was that it deviates significantly from conventional 'intensity-based' assessments [23-28] utilized in seismic design guidelines. Intensity-based assessments seek to determine the seismic response for ground motions with a specific intensity (or specific annual rate of exceedance). This may be the conditional distribution of seismic response for 
$I M=i m, f_{E D P \mid I M}(e d p \mid i m)$, although for simplicity often only the mean response is considered [23-28]. Colloquially, the determination of $f_{E D P \mid I M}(e d p \mid i m)$ for a single IM level is sometimes referred to as a 'stripe analysis' [29], and thus the selection of simulated ground motions for multiple IM levels (or stripes) is herein referred to as 'simple multiple stripes' (the qualifier 'simple' indicates a distinction from Method $4)$.

The results from multiple stripe analyses can be combined with the seismic hazard curve for IM to obtain the seismic demand hazard:

$$
\lambda_{E D P}(e d p)=\sum_{n=1}^{N_{I M}} P_{E D P \mid I M}(e d p \mid i m) \frac{\Delta \lambda_{I M}(\mathrm{im})}{\Delta I M} \Delta I M
$$

where $P_{E D P \mid I M}(e d p \mid i m)$ is the probability that $E D P>e d p$ given $I M=i m ; \Delta I M$ is the increment in IM between the various IM levels considered; and $\Delta \lambda_{I M}(\mathrm{im})$ is the increment in the seismic hazard curve corresponding to $\Delta I M$. Equation (6) is the discrete approximation to the continuous form of the IM-based seismic hazard curve and further details on its computation can be found elsewhere [30, 31].

The algorithm for simulation-based ground motion selection via simple multiple stripes is as follows:

1. Select a conditioning IM to quantify ground motion severity and define a discrete set of $n$ IM levels (not bins, as was used for Method 2).

2. Select $m$ ground motions at each IM level, and perform seismic response analyses to compute $P_{E D P \mid I M}(e d p \mid i m)$

3. Determine the seismic demand hazard based on Equation (6).

It should be noted that in the algorithm above there are two options by which the $m$ ground motions can be selected for $I M=i m$ in step 2. The first is to select the $m$ ground motions whose IM values are closest to $I M=\mathrm{im}$ and use them in seismic response analyses without modification, while the second is to amplitude scale them so that all have exactly $I M=i m$, as is done in GMPE-based ground motion selection $[3,5]$. While the latter option provides some theoretical consistency (given that $P_{E D P \mid I M}(e d p \mid i m)$ requires that $\left.I M=i m\right)$, clearly applying amplitude scaling to (physics-based) simulated ground motions is unappealing because it is unphysical. While the first approach is strictly incorrect in theory, as long as the uncertainty in $E D P \mid I M$ (i.e. the uncertainty in the seismic demands for the given $m$ ground motions) is large relative to the impact of the uncertainty in the $I M$ values of the $m$ selected ground motions (i.e. $\left.\sigma_{\operatorname{lnEDP|IM}} \gg(\partial E D P / \partial I M) \sigma_{\text {lnIM }}\right)$, then $P_{E D P \mid I M}(e d p \mid i m)$ can be estimated by conventional statistical inference with the assumption that $I M \approx i m$ for each ground motion. As shown in the empirical examples below, this assumption is expected to be reasonable in all cases for which the 'set' of available simulated ground motions is large relative to the sub-set which is desired to be selected (i.e. $\left.N_{\text {rup }}^{*} \gg n * m\right)$. On the basis of the above arguments, the use of unmodified ground motion simulations with $I M \approx i m$ is advised to determine $P_{E D P \mid I M}(e d p \mid i m)$, and used in examples herein.

In addition to the manner of selecting $m$ ground motions in step 2 above, $P_{E D P \mid I M}(e d p \mid \mathrm{im})$ can also be estimated by considering the distribution of $E D P \mid I M$ in either a parametric or non-parametric form. Appendix 1 provides a summary of these two possibilities; the non-parametric approach is utilized in the examples below.

Figure 7 provides an example illustration of the application of the simple 
multiple stripes method to quantify the $E D P \mid I M$ relationship through the selection of $m=10$ ground motions at $n=10$ different IM levels; where $S a(\mathrm{~T}=1.32 \mathrm{~s})$ and $P G A$ are utilized as the conditioning IMs. As alluded to earlier, given the large number of available ground motions relative to the sub-set desired (i.e. $N_{\text {rup }}^{*}=5,000 \gg n *$ $m=100$ ) it can be seen that for each of the $n=10$ 'stripes', the uncertianty in EDP is significantly greater than the uncertainty in the IM values of the selected ground motions.

A principal benefit of Method 3 is that it is consistent with 'simple' ground motion selection utilized for GMPE-based SHA (whereas Method 4 is more inline with state-of-the art thinking in this regard). In addition, because the method is IMbased, the seismic response analysis results at each IM level can be used for both 'intensity-based' performance assessments, as well as being integrated with the seismic hazard to obtain the seismic demand hazard curve (i.e. Equation (6)). Furthermore, because the seismic demand hazard may be of interest over only a 'narrow' range of annual exceedance probabilities then it may possible to use as little as, for example, $n=3$ different IM levels [30] allowing for very small numbers of seismic response analyses to be considered (although a parametric approximation of EDP|IM is likely needed in such cases).
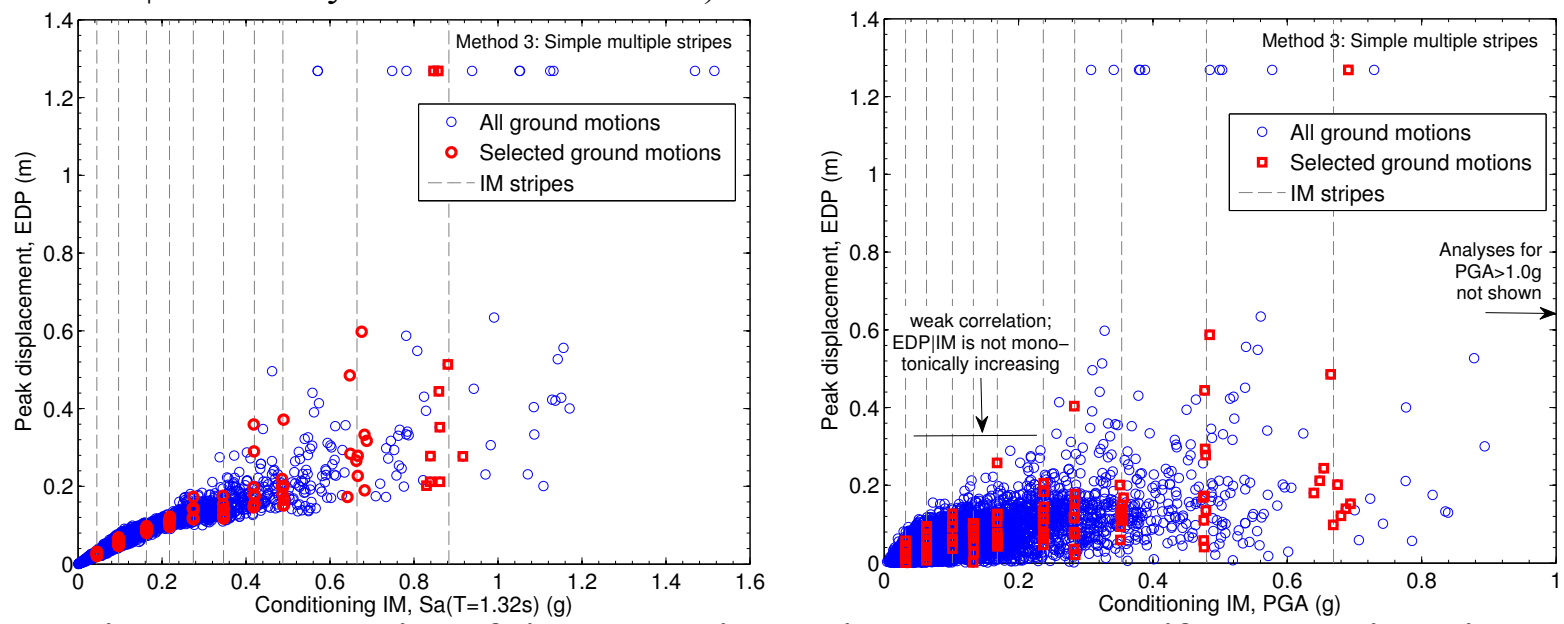

Figure 7: Illustration of simple multiple stripes used to quantify the relationship between EDP and IM through the selection of $m=10$ ground motions at $n=10$ different IM levels (as shown in Figure 1): (a) $I M=S a(T=1.32 s)$; (b) $I M=P G A$.

Despite the benefits mentioned above, Method 3 also suffers from the same problems that exist for the simple 'stripe analysis' approach in conventional GMPEbased ground motion selection. The most important limitation is the strong dependence of the accuracy on the choice of the conditioning IM. Similar to the discussion of Method 2, the greatest accuracy using Method 3 will be obtained when using an 'efficient' conditioning IM which is strongly correlated with the considered EDP (with the aforementioned caveat that no single IM is likely to correlate with a multitude of EDPs which will generally be of interest). In particular, if the selected subset of ground motions for $I M=i m$ are not 'hazard consistent' (in the sense that the distribution of a vector of other intensity measures, $\boldsymbol{I} \boldsymbol{M}$, which the EDP is conditionally dependent on, is different between the sub-set of selected motions and the complete set of motions), then the derived EDP|IM distribution from seismic response analyses will be biased [20, 32-34].

In order to understand its advantages and disadvantages, Figure 8 illustrates the mean and $80 \%$ CI of the seismic demand hazard computed using Method 3, with both 
$I M=S a(T=1.32 s)$ and $I M=P G A$, in comparison to the 'benchmark' Method 1. It can be seen that the result of Method 3 using $I M=S a(T=1.32 s)$ is practically equivalent to Method 1, with a smaller CI than the equivalent Method 2 result in Figure 6. Method 3 using $I M=S a(T=1.32 s)$ is also similar to Method 1 for high frequency exceedance rates, in contrast to the bias seen for Method 2 in Figure 6 (although it is noted that the small 'oscillations' seen for $I M=S a(T=1.32 s$ ) in Figure 8 are the result of the discretization in the non-parametric solution of Equation (6), see Appendix 1). In contrast to the favourable performance using $I M=S a(T=$ $1.32 s$ ), it can be seen that the use of Method 3 with $I M=P G A$ results in a mean and $80 \%$ CI demand hazard which exhibits a notable bias as compared to the Method 1 benchmark, and a wider $C I$ than that using $I M=S a(T=1.32 s)$. As can be seen from Figure $7 \mathrm{~b}$, the correlation between EDP and PGA is such that the subset of $m=10$ selected motions often does not lead to a monotonically increasing mean relationship, and even the use of $m=30$, for example, does not entirely remediate this issue.

One of the contributing factors in the bias in Method 3 using $I M=P G A$ is that the selected ground motions are simply those that have the closest PGA values to the target value at each IM level. Because PGA is such a poor predictor of EDP, then other IM properties of the selected ground motions are significant in determining the seismic response, but are not considered in the selected motions. The consideration of such additional ground motion features beyond a single scalar IM is an improvement considered in Method 4, as discussed in the next section.

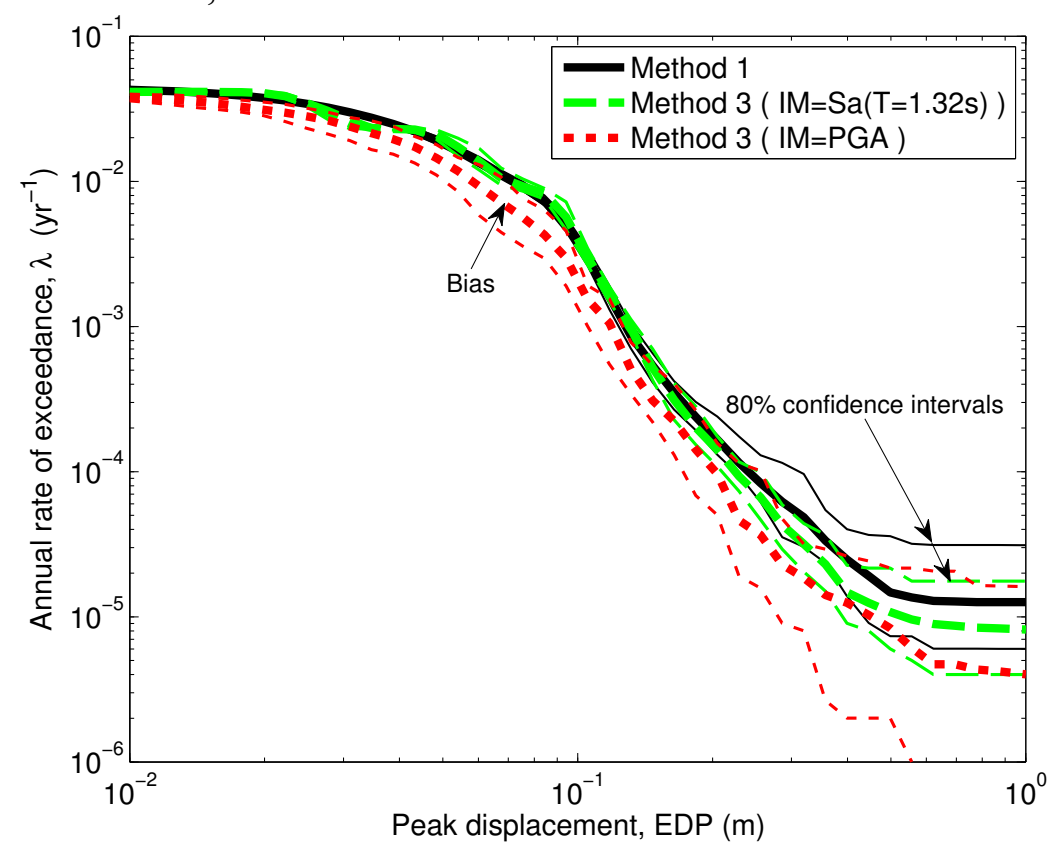

Figure 8: Mean seismic demand hazard curves and $80 \%$ confidence intervals from Method 3 using two different conditioning IMs in comparison with the results from Method 1.

\section{Method 4: GCIM-based selection}

The principal limitation associated with Method 3 is that ground motion selection is solely based on a single conditioning IM, and as a result, a significant amount of information is lost regarding the severity of the selected ground motions (e.g. the importance of directivity-induced velocity pulses, basin-generated surface waves, and near-surface site response, among others, on specific ground motion characteristics that do not impact the conditioning IM). Method 4 for simulation- 
based ground motion selection is based on 'advanced' ground motion selection methods used for GMPE-based SHA. The key idea in this generalized conditional intensity measure (GCIM)-based selection is that ground motions should be selected based on a multitude of different ground motion IMs denoted by the vector $\boldsymbol{I} \boldsymbol{M}$. Since the IM-based computation of the seismic demand hazard (i.e. Equation (6)) uses a single conditioning IM (referred to in this section as $I M_{j}$ for unambiguity) then Method 4 selects a subset of ground motions so that their distribution of $\boldsymbol{I M} \mid I M_{j}$ is statistically indistinguishable from that of the complete set of simulations which defines the seismic hazard at the site. The GCIM-based ground motion selection method [3, 32] (and the specific "conditional spectrum" method [4, 5], when only spectral ordinates are considered within $\boldsymbol{I M}$ ) for GMPE-based SHA are discussed elsewhere, and only their specific variations for simulation-based SHA are elaborated upon here.

The algorithm for simulation-based ground motion selection via the GCIM approach is as follows:

1. Select a conditioning intensity measure, $I M_{j}$, to quantify ground motion severity and define a discrete set of $n$ IM levels.

2. Determine the distribution of $\boldsymbol{I} \boldsymbol{M} \mid I M_{j}$ based on the ground motion simulations which represent the seismic hazard for $I M_{j}=i m_{j}$.

3. Select $m$ ground motions at each $I M_{j}$ level to correctly represent $I \boldsymbol{M} \mid I M_{j}$, and perform seismic response analyses to compute $P_{E D P \mid I M}(e d p \mid i m)$

4. Determine the seismic demand hazard based on Equation (6).

Comparison of the above four step algorithm with that for Method 3 illustrates that they differ only in the computation of $\boldsymbol{I} \boldsymbol{M} \mid I M_{j}$ and specific selection of ground motions to represent this distribution, which are elaborated upon below.

For GMPE-based SHA, Bradley [2, 3] provides the necessary equations for the determination of $\boldsymbol{I} \boldsymbol{M} \mid I M_{j}$ which utilizes empirical GMPEs for each $I M_{i}$ in $\boldsymbol{I} \boldsymbol{M}$ as well as empirical correlation equations between each $I M_{i}$ and $I M_{j}$ [e.g. 35, 36]. In contrast, for simulation-based SHA, neither empirical GMPEs or correlation equations are required because the statistical properties of the appropriate ground motion simulations for $I M_{j}=i m_{j}$ can be directly used to compute $\boldsymbol{I M} \mid I M_{j}$. The only complication is that none of the ground motion simulations will actually exactly have $I M_{j}=i m_{j}$. Similar to the aforementioned application of Method 3, this problem can be practically avoided by utilizing ground motions with $I M_{j} \approx i m_{j}$. In order to do this a lognormal weighting kernel is used to assign weights, $\alpha$, to each of the ground motion simulations. The median of the weighting kernel is fixed at $I M_{j}=i m_{j}$, with a standard deviation of $\sigma_{\operatorname{lnI} M_{j}}$. The kernel is truncated to zero for $I M_{j}$ values exceeding $\pm 3 \sigma_{\operatorname{lnI} M_{j}}$ for computational efficiency (as ground motions beyond this range would have negligible weights in any case). The specific value of $\sigma_{\operatorname{lnI} M_{j}}$ can be considered as somewhat subjective, with a larger value resulting in the consideration of a greater number of ground motion simulations for determining $\boldsymbol{I} \boldsymbol{M} \mid I M_{j}$, yielding more stable distribution statistics, but a greater 'smearing' due to the decreasing applicability of the assumption that $I M_{j} \approx i m_{j}$, and vice versa for a smaller $\sigma_{\operatorname{lnI} M_{j}}$ value. Through sensitivity analyses it was identified that a value of $\sigma_{\operatorname{lnI} M_{j}}=0.05$ consistently provides good results, with negligible sensitivity to $50 \%$ variations in this value, and is generally suggested for use. A value of $\sigma_{\operatorname{lnI} M_{j}}=0.05$ implies that ground motion 
simulations considered in the determination of $\boldsymbol{I} \boldsymbol{M} \mid I M_{j}$ are bounded to be within $15 \%$ (i.e. $\pm 3 \sigma_{\operatorname{lnI} M_{j}}$ ) of $I M_{j}=i m_{j}$, with, on average, $68 \%$ of simulations having $I M_{j}$ values within $5 \%$ of $I M_{j}=i m_{j}$. In comparison with the typical levels of uncertainty in seismic response estimation (e.g. generally $\sigma_{\operatorname{lnEDP|IM}}>0.4[23,37]$ ), the assumption that $I M_{j} \approx i m_{j}$ is thus appropriate, with a negligible effect on the estimation of $\boldsymbol{I M} \mid I M_{j}=i m_{j}$. As a result of the use of the lognormal weighting kernel, for each $I M_{j}$ level of interest, a set of $M$ ground motion simulations will be assigned weights, $\left\{\alpha_{1}, \ldots \alpha_{M}\right\}$, from which the joint distribution of $\boldsymbol{I M} \mid I M_{j}$ can be obtained as elaborated upon in Appendix 2.

An alternative implementation of Method 4 would be to use empirical models for determination of $\boldsymbol{I M} \mid I M_{j}=i m_{j}$ (rather than estimating them from the simulations). This approach would be used if the analyst believed that empirical models for $\boldsymbol{I} \boldsymbol{M}$ were superior to the distribution observed directly from the data [43]; in such a case a set of simulations could still be selected efficiently while also satisfying relevant empirically calibrated IM models. Note that if such an approach is adopted, then Method 1 is no longer a benchmark. This alternative is not further explored in this paper, but may be of practical value in some situations.

Selecting ground motion simulations to represent $\boldsymbol{I} \boldsymbol{M} \mid I M_{j}$ is based on generating random realizations for this multi-variate distribution (see Appendix 2) and then selecting the simulation with the minimum 'misfit', as discussed in references [3, 5]. The relative importance of the various IMs in determining the misfit is quantified using the so-called weight vector, $w_{i}[3]$. Since the simulated ground motions are not amplitude scaled then only ground motions within $\pm 3 \sigma_{l n I M_{j}}$ of $I M_{j}=i m_{j}$ are considered, which is the same constraint used in the construction of the $\boldsymbol{I} \boldsymbol{M} \mid I M_{j}$ distribution.

Figure 9 provides an illustration of $m=10$ selected ground motions using Method 4, for $n=10 I M_{j}=S a(T=1.32 s)$ levels. It can be seen that the selected motions still approximately resemble 'stripes' at each of the $I M_{j}$ levels considered, however it is noted that the motions are no longer simply selected based on those which are closest to $I M_{j}=i m_{j}$. Instead, the ground motions are selected to appropriately represent the distribution $\boldsymbol{I} \boldsymbol{M} \mid I M_{j}$, and an example of the appropriateness of the selected motions in terms of response spectra and 5-95\% significant duration, $D_{s 595}$, is shown in Figure 10. It can be seen in Figure 10a that the empirical $16^{\text {th }}, 50^{\text {th }}$, and $84^{\text {th }}$ percentiles of the selected motions are consistent with the GCIM (i.e. $I \boldsymbol{M} \mid I M_{j}$ ) distributions. Similarly, Figure $10 \mathrm{~b}$ illustrates that the empirical $D_{S 595}$ distribution is consistent with the GCIM distribution based on the Kolmorogov-Smirnov goodness-of-fit criteria. 


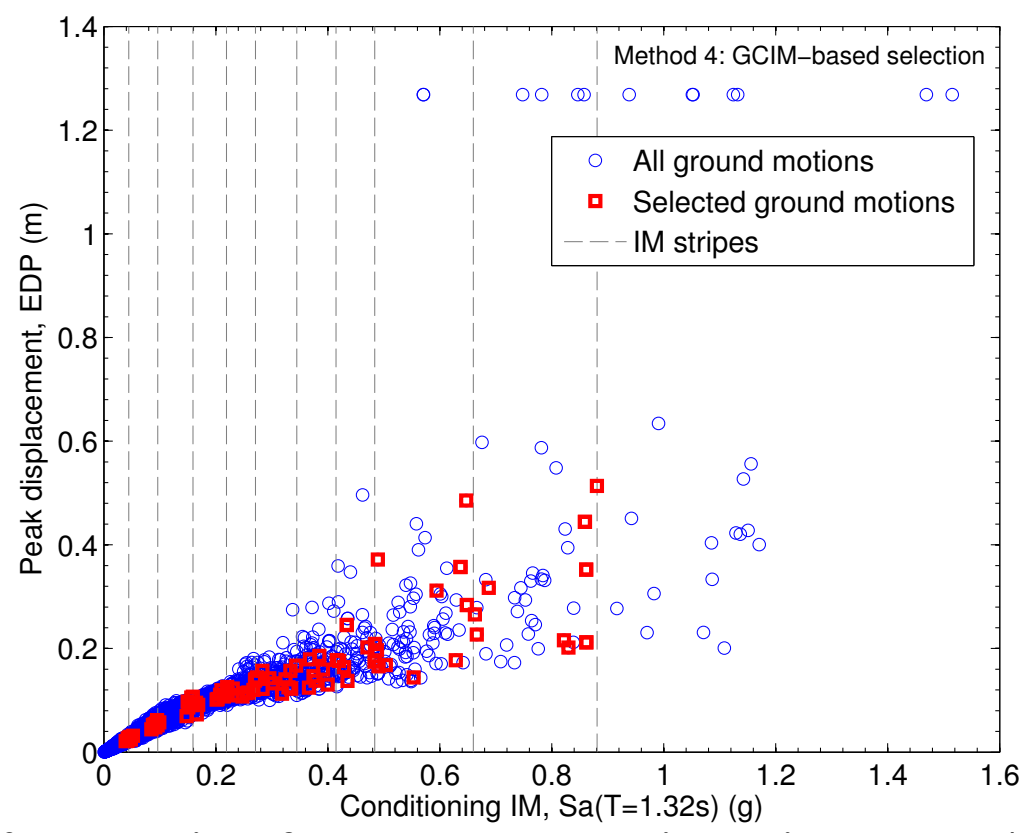

Figure 9: Illustration of selected ground motions using Method 4: GCIM selection for $I M=S a(T=1.32 s)$.
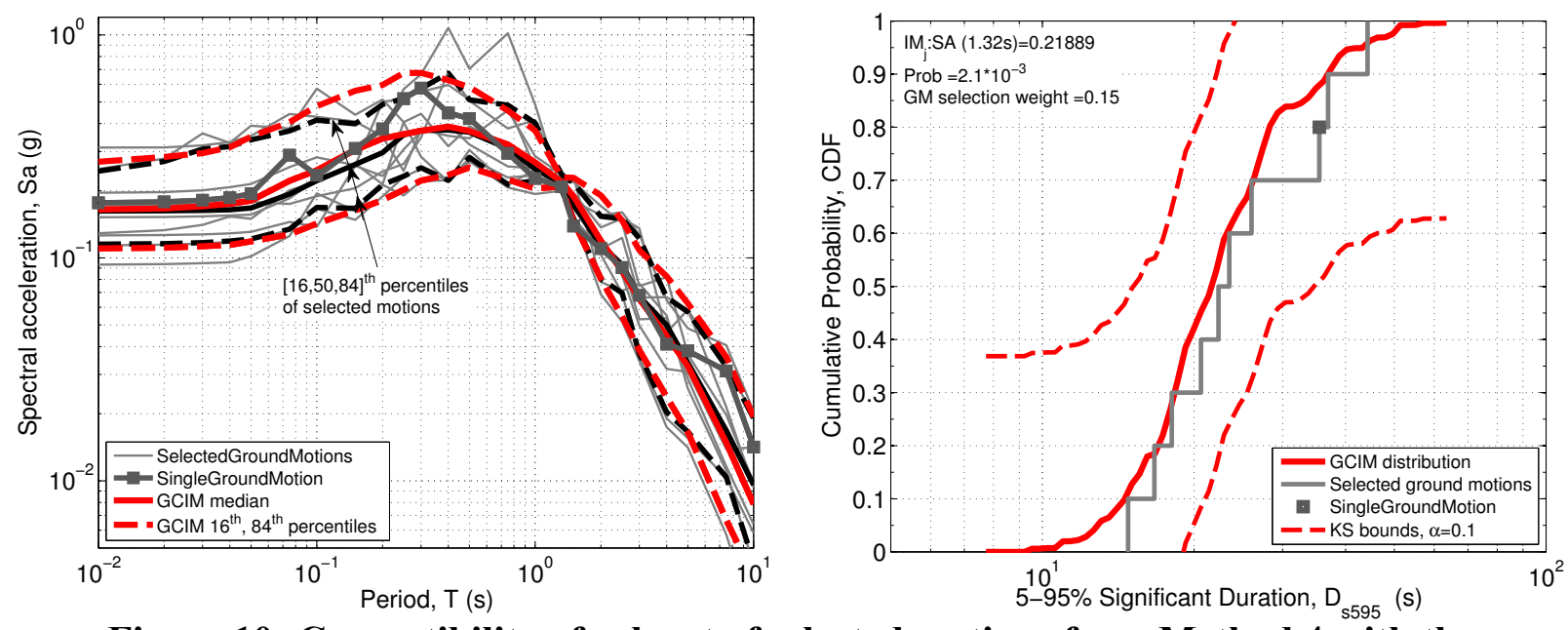

Figure 10: Compatibility of sub-set of selected motions from Method 4 with the $I M \mid I M_{j}$ distribution: (a) response spectra, $S a$; and (b) 5-95\% Significant Duration, $D_{s 595}$.

In comparison with ground motion selection for GMPE-based SHA, obtaining a subset of simulated ground motions which satisfy the target $\boldsymbol{I} \boldsymbol{M} \mid I M_{j}$ distribution is relatively easy, and as a result, the effect of the so-called weight vector, $w_{i}[3]$, is less pronounced. This is because the simulated motions are already site-specific (i.e. simulated for the specific site conditions, and from the specific earthquake sources which dominate the hazard) and have approximately $I M_{j}=i m_{j}$. In contrast, for ground motion selection for GMPE-based SHA, empirical databases of as-recorded ground motions represent a variety of site conditions, from earthquake source and wave propagation in different tectonic and crustal regions, and are amplitude scaled to the target $I M_{j}$ value. Thus, the principal benefit of Method 4 over Method 3 is that selecting ground motions which minimize the misfit with $\boldsymbol{I} \boldsymbol{M} \mid I M_{j}$ will lead to lower variability in the properties of the selected motions, and thus a greater accuracy in the estimated demand relative to Method 3. 
Figure 11 illustrates the mean and $80 \% \mathrm{CI}$ of the seismic demand hazard computed using Method 4, with both $I M_{j}=S a(T=1.32 s)$ and $I M_{j}=P G A$, in comparison to the 'benchmark' Method 1. It can be seen that the results of Figure 11 in summary are very similar to those of Figure 8 . There is no real difference between Method 3 and 4 when $S a(T=1.32 s)$ is used as the conditioning IM, because of its very strong correlation with EDP for this relatively simple structure considered (it would be expected that the benefits of Method 4 would be more prevalent for a more complex system with multiple EDP's of interest). There is a marginal difference between the $I M_{j}=P G A$ result for Method 4 in comparison with that for Method 3 in Figure 8 , most notably a slight reduction in the variability (as indicated by the width of the $\mathrm{CI}$ ) and mean bias for small EDP levels (e.g. $E D P<0.1 \mathrm{~m}$ ). It should also be noted that even with this very poor choice of conditioning IM, the extent of the bias is relatively small, being less than $10 \%$ for $I M_{j}=P G A$ for $\lambda_{E D P}=2.1 * 10^{-3}$ (i.e. $10 \%$ in 50 years exceedance probability). There also exists some bias at very low exceedance probabilities (for both Methods 4 and 3), but as already alluded to, this is a result of a paucity of simulated ground motions from which to choose from at large IM levels.

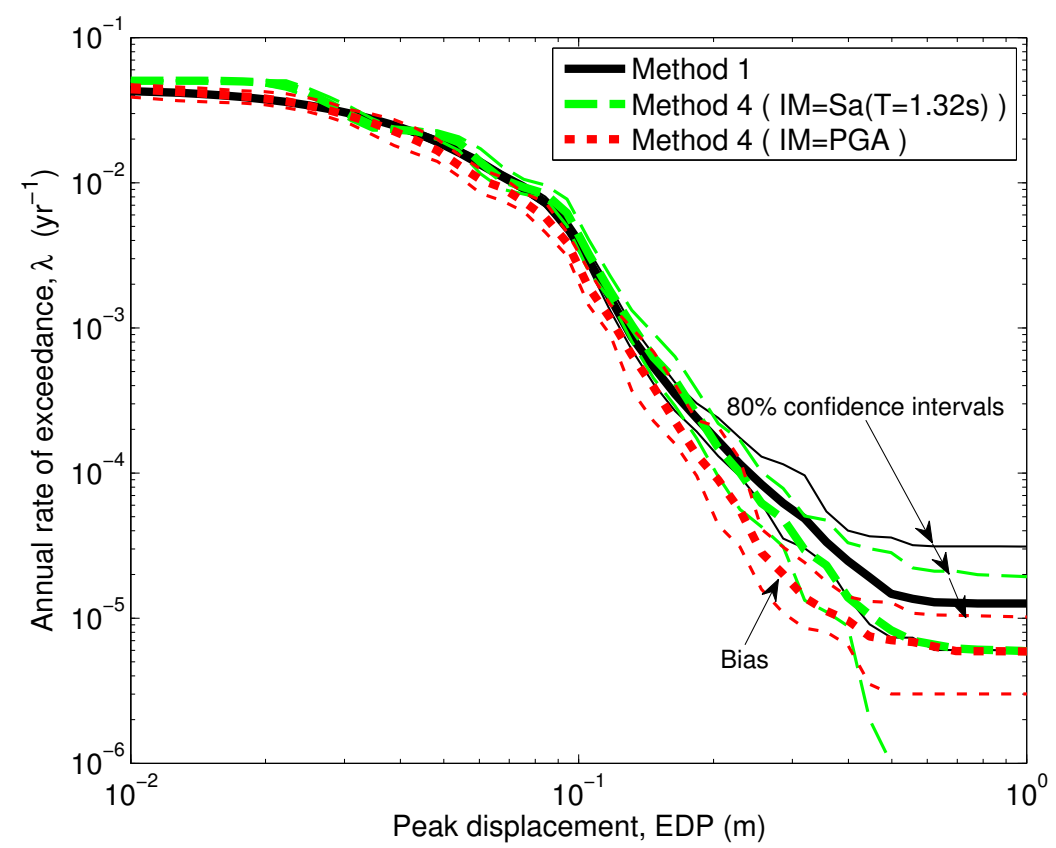

Figure 11: Seismic demand hazard curves from Method 4: GCIM-based selection.

The bias observed in Figure 11 using $I M_{j}=P G A$, although not large in absolute value, was persistent for multiple given ground motion selection weight vectors, and the conditional distributions of the selected ground motions were consistent with the target $\boldsymbol{I} \boldsymbol{M} \mid I M_{j}$ distribution for a large number of IMs (e.g. spectral acceleration, significant durations, Arias intensity, cumulative absolute velocity). This illustrates a limitation of the IM-based approach for very inefficient IMs, where the selected ground motions at each IM level are associated with the incremental exceedance rate, $\Delta \lambda_{I M}(\mathrm{im})$, in contrast to stratified sampling (Method 2) where the selected ground motions are associated with their original rupture rate (and multiplied by an importance sampling factor). Because such an inefficient IM would not be expected to be selected by a knowledgeable analyst, then this example can be considered as a somewhat 'worst-case' scenario. 


\section{DISCUSSION}

The previous section provided detailed discussion and example illustrations of the various advantages and disadvantages of the presented four methods for ground motion selection from simulation-based PSHA. Table 1 provides a summary of these advantages and disadvantages. It is emphasized that these methods are suitable for both PSHA (the focus of illustrations in this paper), and also for scenario-based SHA which is elaborated upon in Appendix 3.

Ground motion selection using Methods 2-4 allow for a significant reduction in the number of seismic response analyses that are required to satisfactorily estimate the demand hazard via the use of a conditioning IM. While $m=10$ ground motions were consider at $n=10 \mathrm{IM}$ levels in each of the examples presented herein (yielding 100 analyses using Methods 2-4, in comparison to 5,000 with Method 1), this number can obviously be specified as a function of the particular precision desired and range of exceedance probabilities of interest. The potential for EDP|IM parametrization in the IM-based approach also allows for the possibility of a very small number of IM levels (e.g. $n=3$ ) to be considered. The accuracy and precision of Methods 2-4 is strongly tied to the 'efficiency' of the conditioning IM to predict the EDP's of interest in the system considered. For a given number of analyses, the IM-based methods (Methods 3 and 4) provide a greater precision. However, Method 2 is unbiased in an average sense irrespective of the 'efficiency' of the conditioning IM, where as some bias may be observed in Methods 3 and 4 when using a very inefficient IM.

The ground motion time series and source codes utilized for performing ground motion selection using the four methods presented in this paper and can be accessed via: http://purl.stanford.edu/wq807vm0009. The open availability of these resources is intended to facilitate implementation of these methods by others and also encourage proponents of alternative simulation-based ground motion selection methods to directly compare such methods with those presented here.

\section{CONCLUSIONS}

This paper examied the selection of simulated ground motion time series for use in seismic response analysis. The availability of site-specific ground motion simulations does not remove the need to be thoughtful in selection of those ground motions for engineering analysis because of the large number of such simulations in relation to the typical numbers of seismic response analysis which are utilized in practice. In this paper, four methods for selecting ground motions from suites of such simulations were presented, as summarized in Table 1. These methods span a range of underlying statistical bases, and/or consistencies with conventional practice for ground motion selection based on ground motion prediction equations and asrecorded motions. The advantages and disadvantages of various methods are discussed, and a simple and reproducible case study utilized to illustrate the methods and provide insights regarding their performance. The data and software underlying the case studies are provided online, for users interested in implementing these approaches or testing the relative performance of their own approach.

\section{ACKNOWLEDGEMENTS}

Constructive comments from Dr. Rob Graves are greatly appreciated. 


\section{REFERENCES}

[1] Bozorgnia, Y, Abrahamson, NA, Atik, LA, Ancheta, TD, Atkinson, GM, Baker, JW, Baltay, A, Boore, DM, Campbell, KW, Chiou, BSJ, Darragh, R, Day, S, Donahue, J, Graves, RW, Gregor, N, Hanks, T, Idriss, IM, Kamai, R, Kishida, T, Kottke, A, Mahin, SA, Rezaeian, S, Rowshandel, B, Seyhan, E, Shahi, S, Shantz, T, Silva, W, Spudich, P, Stewart, JP, Watson-Lamprey, J, Wooddell, K, Youngs, R. NGA-West2 Research Project. Earthquake Spectra 2014; 30(3): 973-987. 10.1193/072113EQS209M

[2] Bradley, BA. A generalized conditional intensity measure approach and holistic ground-motion selection. Earthquake Engineering \& Structural Dynamics 2010; 39(12): 1321-1342. 10.1002/eqe.995

[3] Bradley, BA. A ground motion selection algorithm based on the generalized conditional intensity measure approach. Soil Dynamics and Earthquake Engineering 2012; 40(0): 48-61. 10.1016/j.soildyn.2012.04.007

[4] Baker, JW. The conditional mean spectrum: A tool for ground motion selection. Journal of Structural Engineering 2011; 137(3): 322-331.

[5] Jayaram, N, Lin, T, Baker, JW. A computationally efficient ground-motion selection algorithm for matching a target response spectrum mean and variance. Earthquake Spectra 2011; 27(3): 797-815.

[6] Graves, RW, Pitarka, A. Broadband Ground-Motion Simulation Using a Hybrid Approach. Bulletin of the Seismological Society of America 2010; 100(5A): 2095-2123. 10.1785/0120100057

[7] Mai, PM, Imperatori, W, Olsen, KB. Hybrid Broadband Ground-Motion Simulations: Combining Long-Period Deterministic Synthetics with High-Frequency Multiple S-to-S Backscattering. Bulletin of the Seismological Society of America 2010; 100(5A): 2124-2142. 10.1785/0120080194

[8] Graves, R, Jordan, T, Callaghan, S, Deelman, E, Field, E, Juve, G, Kesselman, C, Maechling, P, Mehta, G, Milner, K, Okaya, D, Small, P, Vahi, K. CyberShake: A Physics-Based Seismic Hazard Model for Southern California. Pure and Applied Geophysics 2011; 168(3): 367-381. 10.1007/s00024010-0161-6

[9] Douglas, J. Consistency of ground-motion predictions from the past four decades. Bulletin of Earthquake Engineering 2010; 8(6): 1515-1526. 10.1007/s10518-010-9195-5

[10] Cornell, CA. Engineering seismic risk analysis. Bulletin of the Seismological Society of America 1968; 58(5): $1583-1606$.

[11] Kramer, SL. Geotechnical earthquake engineering. Prentice-Hall: Upper Saddle River, NJ. 1996; 653.

[12] Field, EH, Gupta, N, Gupta, V, Blanpied, M, Maechling, P, Jordan, TH. Hazard calculations for the WGCEP-2002 forecast using OpenSHA and distributed object technologies. Seismological Research Letters 2005; 76: 161-167.

[13] Jayaram, N, Baker, JW. Statistical tests of the joint distribution of spectral acceleration values. Bulletin of the Seismological Society of America 2008; 98(5): 2231-2243. 10.1785/0120070208

[14] Stirling, M, McVerry, G, Gerstenberger, M, Litchfield, N, Van Dissen, R, Berryman, K, Barnes, P, Wallace, L, Villamor, P, Langridge, R, Lamarche, G, Nodder, S, Reyners, M, Bradley, B, Rhoades, D, Smith, W, Nicol, A, Pettinga, J, Clark, K, Jacobs, K. National Seismic Hazard Model for New Zealand: 2010 Update. Bulletin of the Seismological Society of America 2012; 102(4): 1514-1542. $10.1785 / 0120110170$

[15] Yamamoto, Y, Baker, JW. Stochastic Model for Earthquake Ground Motion Using Wavelet Packets. Bulletin of the Seismological Society of America 2013; 103(6): 3044-3056. $10.1785 / 0120120312$

[16] Ibarra, LF, Medina, RA, Krawinkler, H. Hysteretic models that incorporate strength and stiffness deterioration. Earthquake Engineering \& Structural Dynamics 2005; 34(12): 1489-1511. $10.1002 /$ eqe. 495

[17] Shome, N, Cornell, CA. 1999. Probabilistic seismic demand analysis of nonlinear structures, Stanford University. 357pp.

[18] Cornell, CA, Jalayer, F, Hamburger, RO, Foutch, DA. Probabilistic basis for 2000 SAC federal emergency management agency steel moment frame guidelines. Journal of Structural Engineering 2002; 128(4): 526-533.

[19] Wasserman, L. All of non-parametric statistics. Springer: New York. 2006.

[20] Luco, N, Cornell, CA. Structure-specific scalar intensity measures for near-source and ordinary earthquake ground motions. Earthquake Spectra 2007; 23(2): 357-392. 10.1193/1.2723158

[21] Bradley, BA, Dhakal, RP, Cubrinovski, M, MacRae, GA. Prediction of spatially distributed seismic demands in structures: ground motion and structural response. Earthquake Engineering and Structural Dynamics 2010; 39(5): 501-520. 10.1002/eqe.954

[22] Hastie, T, Tibshirani, R, Friedman, JH. The elements of statistical learning: Data mining, 
inference, and prediction. Springer: New York. 2001; 533.

[23] Bradley, BA. Design Seismic Demands from Seismic Response Analyses: A Probability-Based Approach. Earthquake Spectra 2011; 27(1): 213-224.

[24] ASCE/SEI 7-05. 2006. Minimum design loads for buildings and other structures, American Society of Civil Engineers, ASCE Standard No. 007-05. 388pp.

[25] NZS 1170.5. Structural design actions, Part 5: Earthquake actions - New Zealand. Standards New Zealand: Wellington, New Zealand. 2004; 82.

[26] CEN. 2003. Eurocode 8: Design of structures for earthquake resistance. Part 1: General rules, seismisc actions and rules for buildings, Final Draft prEN 1998, European Committee for Standardization. pp.

[27] FEMA-368. 2001. NEHRP recommended provisions for seismic regulations for new buildings and other structures, 2000 Edition, Part 1: Provisions, Buidling Seismic Safety Council for the Federal Emergency Management Agency. pp.

[28] Bradley, BA. A comparison of intensity-based demand distributions and the seismic demand hazard for seismic performance assessment. Earthquake Engineering \& Structural Dynamics 2013; 42(15): 2235-2253. 10.1002/eqe.2322

[29] Jalayer, F. Direct probabilistic seismic analysis: Implementing non-linear dynamic assessments, in Department of Civil and Environmental Engineering. Stanford University: Stanford, CA. 2003; 173 pp. [30] Bradley, BA. Practice-oriented estimation of the seismic demand hazard using ground motions at few intensity levels. Earthquake Engineering \& Structural Dynamics 2013; 42(14): 2167-2185. 10.1002/eqe. 2319

[31] Bradley, BA, Lee, DS, Broughton, R, Price, C. Efficient evaluation of performance-based earthquake engineering equations. Structural Safety 2009; 31(1): 65-74. 10.1016/j.strusafe.2008.03.003 [32] Bradley, BA. A generalized conditional intensity measure approach and holistic ground motion selection. Earthquake Engineering and Structural Dynamics 2010; 39(12): 1321-1342. 10.1002/eqe. 995

[33] Baker, JW. Vector-valued ground motion intensity measures for probabilistic seismic demand analysis, in Department of Civil and Environmental Engineering. Stanford University: California. $2005 ; 347$.

[34] Bradley, BA. The seismic demand hazard and importance of the conditioning intensity measure. Earthquake Engineering \& Structural Dynamics 2012; 41(11): 1417-1437. 10.1002/eqe.2221

[35] Baker, JW, Jayaram, N. Correlation of spectral acceleration values from NGA ground motion models. Earthquake Spectra 2008; 24(1): 299-317.

[36] Bradley, BA. Correlation of Significant Duration with Amplitude and Cumulative Intensity Measures and Its Use in Ground Motion Selection. Journal of Earthquake Engineering 2011; 15(6): 809-832. 10.1080/13632469.2011.557140

[37] Aslani, H, Miranda, E. Probability-based Seismic Response Analysis. Engineering Structures 2005; 27(8): 1151-1163.

[38] Aslani, H, Miranda, E. Fragility assessment of slab-column connections in existing non-ductile reinforced concrete buildings. Journal of Earthquake Engineering 2005; 9(6): 777-804.

[39] Ramirez, CM, Miranda, E. 2009. Building-specific loss estimation methods and tools for simplified performance-based earthquake engineering, Stanford University, Blume Centre report number $171.370 \mathrm{pp}$.

[40] Mackie, KR, Stojadinovic, B. Performance-based seismic bridge design for damage and loss limit states. Earthquake Engineering and Structural Dynamics 2007; 36(13): 1953-1971. 10.1002/eqe.699

[41] Graves, RW, Aagaard, BT, Hudnut, KW. The ShakeOut Earthquake Source and Ground Motion Simulations. Earthquake Spectra 2011; 27(2): 273-291. 10.1193/1.3570677

[42] Melchers, RE. Structural reliability analysis and prediction. John Wiley and Sons: Chichester. 1999.

[43] Burks, LS, Baker, JW. Validation of ground motion simulations through simple proxies for the response of engineered systems. Bulletin of the Seismological Society of America 2015; (in press).

\section{APPENDIX 1: PARAMETRIC AND NON-PARAMETRIC COMPUTATION OF THE IM-BASED DEMAND HAZARD INTEGRAL}

The IM-based computation of the demand hazard (Equation (6)) requires 
summation (i.e. numerical integration) over a range of IM values, which can be obtained using both parametric and non-parametric approaches.

\section{Non-parametric computation}

The summation in the seismic demand hazard, as given by Equation (6), is considered over the $n$ IM levels for which ground motions are selected. For each IM level, the $E D P \mid I M$ exceedance probability is computed from the fraction of the $m$ ground motions which exceed $E D P=e d p$ :

$$
P_{E D P \mid I M}(e d p \mid i m)=\frac{1}{m} \sum_{p=1}^{m} I\left(E D P_{p}>e d p\right)
$$

where $E D P_{p}$ is the seismic demand from the $\mathrm{p}^{\text {th }}$ ground motion. The increment in the seismic demand hazard, $\Delta \lambda_{I M}(\mathrm{im})$, is obtained as the increment between the midpoints of $\lambda_{I M}$ values above and below the specific IM level of interest. After algebraic manipulation, this increment can be expressed as:

$$
\Delta \lambda_{I M}\left(i m_{p}\right)=\frac{1}{2}\left[\lambda_{I M}\left(i m_{p-1}\right)-\lambda_{I M}\left(i m_{p+1}\right)\right]
$$

where the subscript $p$ in Equation (8) indicates one of the $n$ IM levels. For the first IM level $(p=1)$, the hazard increment can be computed as $\Delta \lambda_{I M}\left(i m_{1}\right)=\left[\lambda_{I M}\left(i m_{1}\right)-\lambda_{I M}\left(i m_{2}\right)\right]$; similarly for the last IM level $\Delta \lambda_{I M}\left(i m_{n}\right)=$ $\left[\lambda_{I M}\left(i m_{n-1}\right)-\lambda_{I M}\left(i m_{n}\right)\right]$. Equation (8) and those for the first and last IM levels correspond to classic central, forward, and backward differences.

While the non-parametric evaluation of the seismic demand hazard requires minimal information in the definition of $P_{E D P \mid I M}(e d p \mid i m)$, and summation over only $n$ IM levels, this summation can result in appreciable errors; namely: (i) near the maximum and minimum IM levels considered; and (ii) if the 'spacing' between the considered IM levels is significant. In such cases a parametric approximation may be more appropiate.

\section{Parametric computation}

There are various ways in which $P_{E D P \mid I M}(e d p \mid i m)$ can be parametrized, and attention here is restricted to summarizing the approach in Section 4 of Bradley [30]. It is generally reasonable to assume that the distribution of $P_{E D P \mid I M}(e d p \mid i m)$ is lognormal [e.g. 17, 38, 39], with distribution parameters (mean and variance) estimated from the $m$ seismic response results at each of the $n$ IM levels. Previous parametric functions for the central-tendency of the EDP $\mid I M$ relationship have often been of a power-model form, such that the relationship between $\ln (E D P)$ and $\ln (I M)$ is linear [e.g. 18, 37, 40]. Therefore a piece-wise power-model function can be used to describe the lognormal mean, $\mu_{\operatorname{lnEDP|IM}}$, between each of the IM levels considered (Equation 2 in [30]). Some extrapolation of the EDP $I M$ relationship is possible if the piecewise function over the first and last IM intervals is simply extended. In a similar manner, the lognormal standard deviation, $\sigma_{\operatorname{lnEDP|IM}}$, can be expressed using a piecewise linear function between the $n$ IM levels (Equation 3 in [30]), and extrapolated as a constant value beyond the minimum and maximum IM levels.

With $P_{E D P \mid I M}(e d p \mid i m)$ parametrically represented continuously over the range of IM levels considered (as well as reasonable amount of extrapolation), Equation (6) 
can be evaluated via summation over a large number of IM values, allowing for a more accurate representation of the derivate of the hazard curve $\left(\Delta \lambda_{I M}(\mathrm{im}) / \Delta I M\right)$, and reducing inaccuracies at the exceedance rates corresponding to the maximum and minimum IM levels by allowing for an appropiate level of extrapolation [30].

\section{APPENDIX 2: EMPIRICAL MULTI-VARIATE DISTRIBUTION OF A WEIGHTED MULTI-VARIATE DATA SAMPLE}

Method 4 utilizes the multi-variate distribution, $\boldsymbol{I M} \mid I M_{j}$, which is obtained from a weighted sample of the IMs from $M$ ground motion simulations, $\left\{\boldsymbol{i m}_{1}, \ldots \boldsymbol{i} \boldsymbol{i m}_{p}, \ldots \boldsymbol{i} \boldsymbol{i m}_{M}\right\}$ (where each sample, e.g. $\boldsymbol{i m}_{p}$, is a vector of different ground motion intensity measures), with corresponding normalized weights, $\left\{\alpha_{1}, \ldots \alpha_{p}, \ldots \alpha_{M}\right\}$; where $\Sigma \alpha=1$. The calculations for the marginal distributions and correlation coefficients of this multi-variate distribution, and random number generation are presented below.

\section{Empirical marginal distribution}

For each of the ground motion IMs of interest in the vector $\boldsymbol{I M}$, the nonparametric marginal distribution can be obtained from:

$$
F_{I M_{i} \mid I M_{j}}(x)=\sum_{p=1}^{M} \alpha_{p} * I\left(x>\operatorname{im}_{i, p}\right)
$$

where $F_{I M_{i} \mid I M_{j}}$ is the (empirical) cumulative distribution function (CDF) of $I M_{i} \mid I M_{j}$, and $i m_{i, p}$ is the value of $I M_{i}$ for the $\mathrm{p}^{\text {th }}$ ground motion.

\section{Correlation}

In order to generate correlated random numbers from $\boldsymbol{I} \boldsymbol{M} \mid I M_{j}$ it is necessary to know the correlation between each of the IMs in $\boldsymbol{I} \boldsymbol{M}$. While the non-parametric empirical distributions are directly used in this random number generation, the empricial distributions are assumed to be 'lognormal-like' so that the Pearson correlation coefficeint between the logarithm of the two different IMs, $I M_{i}$ and $I M_{k}$ (from $\boldsymbol{I M}$ ) provides a suitable correlation metric, and can be computed from:

$$
\rho_{\text {lnI } M_{i}, \operatorname{lnI} M_{k} \mid I M_{j}}=\frac{\sigma_{i, k}}{\sqrt{\sigma_{i, i} \sigma_{k, k}}}
$$

where

$$
\begin{aligned}
& \sigma_{i, k}=\sum_{p=1}^{M} \alpha_{p}\left[\ln \left(i m_{i, p}\right)-\hat{\mu}_{l n I M_{i} \mid I M_{j}}\right]\left[\ln \left(i m_{k, p}\right)-\hat{\mu}_{l n I M_{k} \mid I M_{j}}\right] \\
& \hat{\mu}_{l n I M_{i} \mid I M_{j}}=\sum_{p=1}^{M} \alpha_{p} * \ln \left(i m_{i, p}\right)
\end{aligned}
$$

\section{Random number generation}

Because the distribution of $\boldsymbol{I} \boldsymbol{M} \mid I M_{j}$ is non-parametric then correlated random number generation can be performed by using the inverse transformation method [19]. Firstly, correlated random numbers are generated from a multi-variate normal 
distribution with unit variances and transformed to variates with marginal uniform distributions through the inverse normal transform:

$$
\boldsymbol{U}=\Phi^{-1}[\operatorname{MVN}(\mathbf{0}, \boldsymbol{\rho})]
$$

where $\boldsymbol{U}$ is a vector of correlated random numbers with marginally-uniform distributions; $\Phi^{-1}$ is the inverse normal distribution function; and $\operatorname{MVN}(\mathbf{0}, \boldsymbol{\rho})$ is a multi-variate normal distribution with zero mean and covariance matrix equal to the correlation matrix (i.e. all variances are unity). The random values of each $I M_{i}$ in $\boldsymbol{I M}$ can then be obtained from the inverse transformation method by using the CDF for $I M_{i}$ :

$$
i m_{i}=F_{I M_{i} \mid I M_{j}}^{-1}\left(U_{i}\right)
$$

where $U_{i}$ is the $i^{\text {th }}$ element of $\boldsymbol{U}$. The application of Equation (9) for all $I M_{i}$ 's in $\boldsymbol{I M}$ yields the desired random vector.

\section{APPENDIX 3: GROUND MOTION SELECTION FOR SCENARIO SEISMIC HAZARD ANALYSIS (SCENARIO-BASED SHA)}

In addition to the use of ground motion simulations to determine the seismic demand hazard, it is often also of interest to understand the distribution of seismic response for a scenario earthquake rupture, $P_{E D P \mid R u p}\left(e d p \mid r u p_{k}\right)$. The $M_{w} 7.8$ ShakeOut scenario [41] is one example; however, the distribution of seismic response considering variability in the hypocenter, slip and time evolution are also of interest, in contrast to the single prescriptions used for ShakeOut. In order to adequately sample this variability it make be necessary to randomly generate $N_{k}$ ground motion simulations to represent the rupture which are greater than that feasible for seismic response analysis - hence the need for ground motion selection.

The approach of ground motion selection Method 1 is to consider all $N_{k}$ simulations to determine $P_{E D P \mid R u p}$. By considering a conditioning IM, stratified sampling (Method 2) can be used in the same manner to preferentially sample the tails of the $I M \mid R u p$ distribution, and thus efficiently estimate the EDP $\mid R u p$ distribution when an efficient IM is utilized. While it is possible to obtain the EDP $\mid R u p$ distribution via summation over an IM intermediatery variable (i.e. equivalent to Method 3) this approach is not very appealing, because the conditional distribution, $E D P \mid R u p, I M$ is not of general interest. Finally, a scenario-based version of Method 4 involves the determination of the multivariate distribution, $\boldsymbol{I} \boldsymbol{M} \mid$ Rup , in the same manner as for the PSHA-case (i.e. Appendix 2, with each of the $N_{k}$ simulations having an equal weight of $\left.\alpha_{p}=1 / N_{k, p}\right)$; and then ground motion selection via those simulations with the minimum misfit to random realizations from IM|Rup. Similar to Method 2, this scenario-based application of Method 4 will lead to a more efficient determination of the EDP $\mid$ Rup distribution by selecting a smaller subset of motions with IM values which are representative of the overall ground motion simulation set. 
Table 1: Summary of simulation-based ground motion selection methods for estimation of the seismic demand hazard

\begin{tabular}{|c|c|c|c|}
\hline Method & Concept & Pros & Cons \\
\hline 1. Direct analysis & $\begin{array}{l}\text { Consider every ground motion in } \\
\text { seismic hazard calculation }\end{array}$ & $\begin{array}{c}\text { 'Exact' solution } \\
\text { Conceptually simple, no conditional } I M \text { needed }\end{array}$ & $\begin{array}{l}\text { Computational demanding (often prohibitively) } \\
\text { Inefficient: most seismic response analyses are performed on frequent, but } \\
\text { low-amplitude motions, providing little information about response levels } \\
\text { of engineering interest }\end{array}$ \\
\hline 2. Stratified sampling & $\begin{array}{l}\text { Bin ground motions based on } \\
\text { conditioning } I M \text { values; select } \\
\text { subsets from each bin }\end{array}$ & $\begin{array}{l}\text { Relatively simple } \\
\text { Will result in a greater proportion of high-amplitude ground motions } \\
\text { compared to Method 1; specific amplitudes of interest can be targeted via IM } \\
\text { bin selection } \\
\text { IM hazard curve not directly required; implicitly captured via the } \\
\text { renormalized rates of the selected ground motions }\end{array}$ & $\begin{array}{l}\text { Accuracy and precision strongly dependent on 'efficiency' of conditioning } \\
\text { IM to predict the multiple EDPs of interest } \\
\text { Choice of bin width maybe affect results in a non-trivial manner }\end{array}$ \\
\hline $\begin{array}{l}\text { 3. Simple multiple } \\
\text { stripes }\end{array}$ & $\begin{array}{l}\text { Select ground motions for specific } \\
I M=i m \text { values. Develop } \\
\text { parametric expression for } \\
\qquad E D P \mid I M\end{array}$ & $\begin{array}{l}\text { Consistent with conventional practice using as-recorded ground motions } \\
\text { Interpolation as a function of } I M \text { allows for reduction in analysis, as well as } \\
\text { parametric interpretation of results such as 'intensity-based' performance } \\
\text { assessments }\end{array}$ & $\begin{array}{l}\text { Limited to consideration of a scalar } I M \text {, thus 'throwing away' potential } \\
\text { information on ground motion severity } \\
\text { Demand hazard dependent on the manner in which } E D P \mid I M \text { is } \\
\text { parameterized and the integral evaluated (important for few IM levels) }\end{array}$ \\
\hline $\begin{array}{l}\text { 4. GCIM-based } \\
\text { selection }\end{array}$ & $\begin{array}{l}\text { Same as Method 3, except that } \\
\text { ground motions selected so that } \\
\text { the distribution of a vector of IMs, } \\
\boldsymbol{I M}, \text { is also consistent with } \\
\text { seismic hazard }\end{array}$ & $\begin{array}{c}\text { All the benefits of Method } 3 \\
\text { Consistent with GCIM/CS ground motion selection approaches for as- } \\
\text { recorded ground motions } \\
\text { Don't need to pick a single scalar } I M \\
\text { More robustly captures record properties not measured by scalar } I M \text {, even } \\
\text { with small sets of } m \text { ground motions }\end{array}$ & $\begin{array}{l}\text { Determination of selected ground motions significantly more involved than } \\
\text { Methods } 1-3 \\
\text { Demand hazard dependent on the manner in which EDP } \mid I M \text { is } \\
\text { parameterized and the integral evaluated (important if only a few IM levels } \\
\text { are considered) }\end{array}$ \\
\hline
\end{tabular}

